\title{
Soft optically tuneable fluorescence phantoms based on gel wax and quantum dots: a tissue surrogate for fluorescence imaging validation
}

Yijing Xie, Efthymios Maneas, Shahraz Islam, William Peveler, Jonathan Shapey, et al.

Yijing Xie, Efthymios Maneas, Shahraz Islam, William Peveler, Jonathan Shapey, Wenfeng Xia, Sebastien Ourselin, Ivan P. Parkin, Adrien E. Desjardins, Tom Vercauteren, "Soft optically tuneable fluorescence phantoms based on gel wax and quantum dots: a tissue surrogate for fluorescence imaging validation," Proc. SPIE 10862, Molecular-Guided Surgery: Molecules, Devices, and Applications V, 108621F (7 March 2019); doi: 10.1117/12.2508348 


\title{
Soft optically-tuneable fluorescence phantoms based on gel wax and quantum dots: a tissue surrogate for fluorescence imaging validation
}

\author{
Yijing Xie ${ }^{\mathrm{a}, \mathrm{b}} *$, Efthymios Maneas ${ }^{\mathrm{b}, \mathrm{c}}$, Shahraz Islam ${ }^{\mathrm{b}}$, William Peveler ${ }^{\mathrm{d}, \mathrm{e}}$, Jonathan Shapey ${ }^{\mathrm{c}}$, Wenfeng \\ $\mathrm{Xia}^{\mathrm{a}, \mathrm{b}}$, Sebastien Ourselin ${ }^{\mathrm{a}}$, Ivan P. Parkin ${ }^{\mathrm{d}}$, Adrien E. Desjardins, ${ }^{\mathrm{b}, \mathrm{c}}$, Tom Vercauteren ${ }^{\mathrm{a}}$

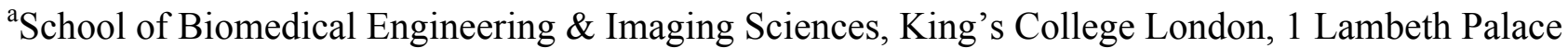 \\ Road, London SE1 7EU, United Kingdom; \\ ${ }^{b}$ Department of Medical Physics and Biomedical Engineering, University College London, Gower \\ Street, London WC1E 6BT, United Kingdom; \\ ${ }^{c}$ Wellcome/EPSRC Centre for Interventional and Surgical Science, University College London, 43 \\ Foley Street, London W1W 7TS, United Kingdom \\ ${ }^{\mathrm{d}}$ Department of Chemistry, University College London, 20 Gordon Street, London WC1H OAJ, \\ United Kingdom; \\ ${ }^{\mathrm{e}}$ Division of Biomedical Engineering, University of Glasgow, Oakfield Ave., Glasgow G12 8LT, \\ United Kingdom; \\ *yijing.xie@kcl.ac.uk
}

\begin{abstract}
Fluorescence-guided brain tumour resection, notably using 5-aminolevulinic acid (ALA)-induced protoporphyrin IX (PpIX) for high-grade gliomas, has been demonstrated to provide better tissue differentiation, thereby improving patient outcomes when compared to white-light guidance. Novel fluorescence imaging devices aiming to increase detection specificity and sensitivity and targeting applications beyond high-grade gliomas are typically assessed by measurements using tissue-mimicking optical phantoms. The field currently lacks adequate phantoms with well-characterised tuneable optical properties. In this study, we developed soft tissue-mimicking fluorescence phantoms (TMFP) highly suitable for this purpose. We investigated: 1) the ability to independently tune optical and fluorescent properties; 2 ) the stability of the fluorescence signal over time; and 3 ) the potential of the proposed phantoms for imaging device validation. The TMFP is based on gel-wax which is an optically transparent mineral-oil based soft material. We embedded $\mathrm{TiO}_{2}$ as scattering material, carbon black oil-paint as background absorber, and CdTe Quantum Dots (QDs) as fluorophore because of its similar fluorescence spectrum to PpIX. Scattering and absorption properties were measured by a spectrophotometer, while the fluorescence was assessed by a wide-field fluorescence imaging system (WFFI) and a spectrometer. We demonstrated that: 1) the addition of QDs didn't alter the phantom's scattering which was only defined by the concentration of TiO2, whereas its absorption was defined by both QDs and colour oil paint; 2) the measured fluorescence intensity was linearlyproportional to the concentration of QDs; 3 ) the fluorescence intensity was stable over time (up to eight months); and 4) the fluorescence signal measured by the WFFI were strongly correlated to spectrometer measurements.
\end{abstract}

Keywords: fluorescence imaging, tissue-mimicking phantoms, gel wax, quantum dots, brain tumour resection

\section{INTRODUCTION}

Wide field fluorescence imaging with 5-ALA-PpIX has been promising to guide brain tumour resection by providing superior tissue differentiation as compared to white-light guidance [1-3]. The development and clinical translation of novel fluorescence imaging devices require tissue-mimicking phantoms for standardised performance evaluation [4]. Phantoms for fluorescence imaging need to have three basic requirements. First, the optical properties (optical absorption, scattering and fluorescence) of the phantom need to be tuneable so that they can simulate the optical properties of a variety of soft

Molecular-Guided Surgery: Molecules, Devices, and Applications V, edited by Brian W. Pogue, Sylvain Gioux, Proc. of SPIE Vol. 10862, 108621F · @ 2019 SPIE · CCC code: 1605-7422/19/\$18 · doi: 10.1117/12.2508348 
tissues. Second, the developed phantom needs to be mechanically and optically stable over time. Last, the phantom needs to be able to simulate the anatomical features of the organ (brain) for imaging system validation $[5,6]$.

Many materials have been used for tissue mimicking phantoms for optical imaging, however, a durable and reliable phantom for fluorescence imaging is highly desired. Intralipid, agar and gelatine have been widely used for optical phantoms, however, they are not mechanically stable [7]. Polyester resin phantoms are mechanically strong, but they do not mimic the mechanical properties of soft tissues [8]. Gel wax is a non-toxic soft tissue mimicking material that has recently shown great promise for phantoms in optical and ultrasound imaging [9-10]. Its mechanical, optical and acoustic properties can be tuned to mimic human soft tissues.

In this study, we introduce soft tissue-mimicking fluorescence phantoms (TMFP) based on gel wax and quantum dots. We investigated: 1) the ability to independently tune the scattering, absorption and fluorescence of the phantoms; 2) the stability of the TMFP fluorescence signal over time; and 3) the potential of the proposed phantoms for the validation of imaging systems.

\section{MATERIALS AND METHODS}

\subsection{Phantom fabrication}

The composite phantom comprised 8 inclusions in a background matrix based on gel wax. Native gel wax is a mineral oilbased, transparent, and soft material (Figure 1(a)) [10]. Its optical absorption and scattering can be controlled with Carbo black ink and Titanium Dioxide as additives, respectively (Figure 1(b)). The material can be moulded to mimic anatomically realistic tissue features and is mechanically robust as shown in Figure 1(c-d). CdTe quantum dots (QDs) are chosen as fluorophore to resemble 5-ALA-PpIX, as they share similar excitation and emission spectra (Figure 2). QDs hold many advantages over organic fluorophores which are often used in phantom preparations, such as high absorption coefficient across a wide spectral range, tuneable fluorescence emission and narrow emission spectra, and photostability [5,11]. The material handling and manufacturing details can be found in works of Maneas et al. [9-10]. Two sets of inclusions were fabricated: the first set (A11, A12, A13, A14) comprised 4 inclusions with a carbon black ink concentration of $0.050 \mathrm{~g} / \mathrm{mL}$, a titanium dioxide concentration of $0.075 \mathrm{~g} / \mathrm{mL}$, and varying quantum dots concentrations from 0.001 to $0.010 \mathrm{v} / \mathrm{v} \%$. The second set comprised 4 inclusions with the same the same carbon black ink and quantum dots concentration concentrations as for the first set, but with a higher titanium dioxide concentration $(0.010 \mathrm{~g} / \mathrm{mL})$ (Table 1).
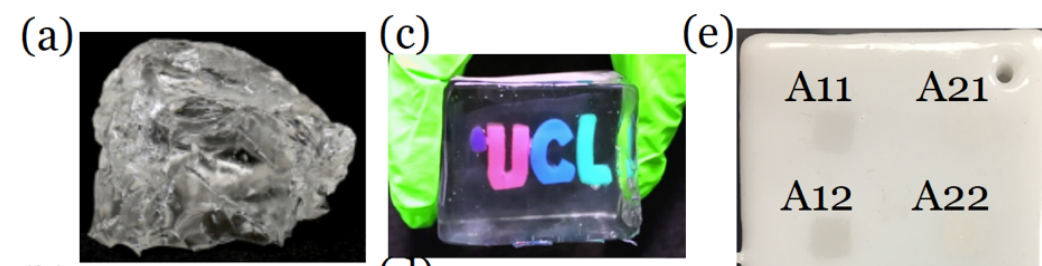

(b)
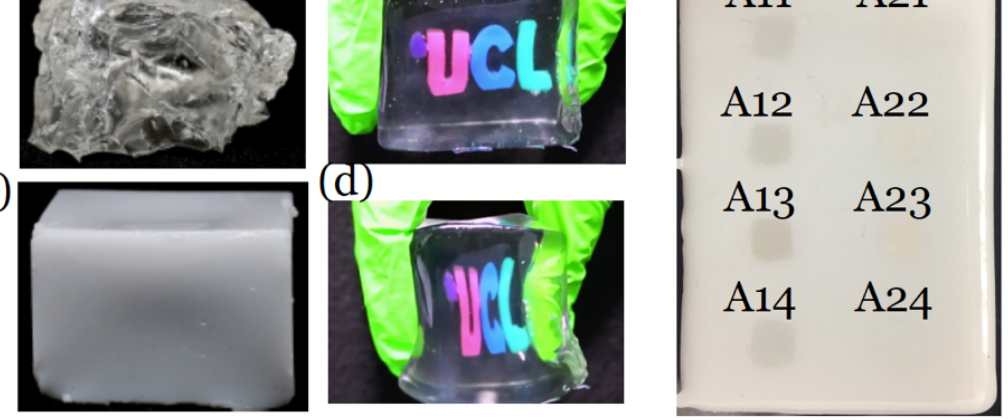

Figure 1. (a) Native gel wax, (b) Native gel wax with TiO2 and Carbon Black ink as additives, (c, d) Squashing a gel wax phantom with inclusions having complex geometry (e) The composite phantom. (a-d) are adopted from Ref [10] with permission.

Table 1. Compositions of the phantom batches

\begin{tabular}{|c|c|c|c|}
\hline Batches & $\begin{array}{l}\text { Carbon black ink } \\
\text { concentration }(\mathrm{g} / \mathrm{mL})\end{array}$ & $\begin{array}{l}\text { Titanium dioxide } \\
\text { concentration }(\mathrm{g} / \mathrm{mL})\end{array}$ & $\begin{array}{l}\text { Quantum dots } \\
\text { concentration (v/v\%) }\end{array}$ \\
\hline A11, A12, A13, A14 & 0.050 & 0.075 & $0.001,0.003,0.006,0.010$ \\
\hline $\mathrm{A} 21, \mathrm{A22}, \mathrm{A23}, \mathrm{A24}$ & 0.050 & 0.100 & $0.001,0.003,0.006,0.010$ \\
\hline
\end{tabular}



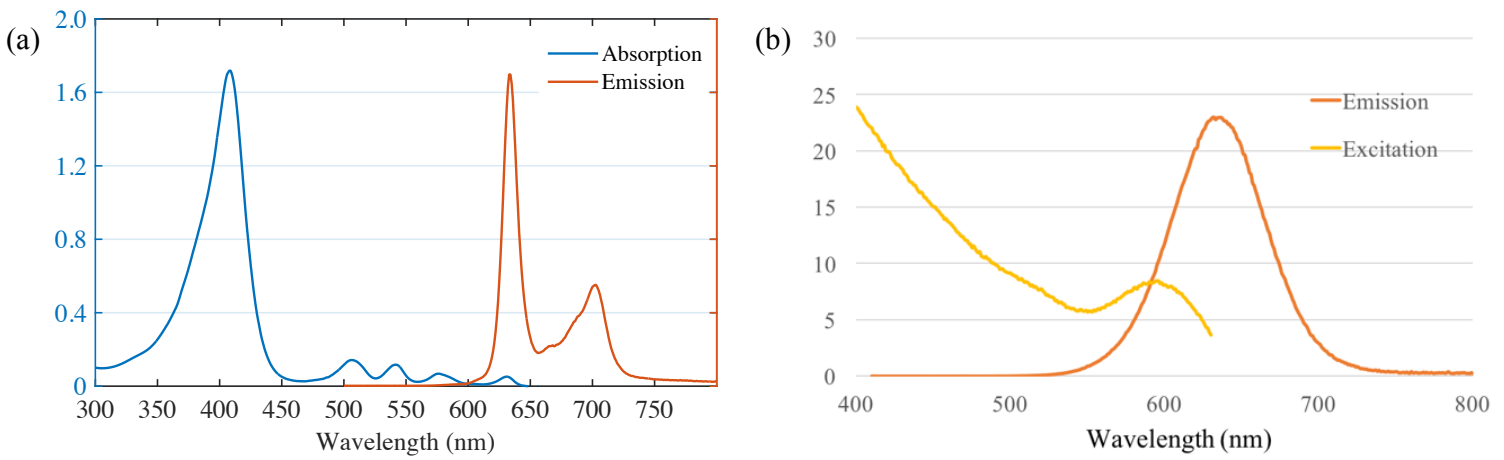

Figure 2: The excitation and emission spectra of PpIX (a) and CdTe quantum dots (b).

\subsection{Characterisation of optical absorption and scattering properties}

The optical absorption and scattering spectra of each phantom batch was determined using a spectrophotometer with an integration sphere (Lambda 750, Perkins Elmer). For each phantom batch, a thin phantom slab with dimension of $35 \mathrm{~mm}$ (W) $\times 50 \mathrm{~mm}$ (L) $\times 2 \mathrm{~mm}(\mathrm{D})$ was fabricated and the reflectance (R) and total transmittance spectra (T) of the phantom slab was measured with the spectrophotometer. An inverse adding doubling algorithm [12] was used to estimate the optical absorption $\left(\mu_{\mathrm{a}}\right)$ and reduced scattering $\left(\mu_{\mathrm{s}}{ }^{\prime}\right)$ spectra from $\mathrm{R}$ and T for a wavelength range of $400-1600 \mathrm{~nm}$.

\subsection{Characterisation of temporal stability of phantom's fluorescent signal}

To characterise the temporal stability of the phantom's fluorescent signal, the slab phantoms were excited with an LED light source at $405 \mathrm{~nm}$ with a power of $17 \mathrm{~mW}$. The fluorescent signal intensity for each slab was measured by a compact CCD spectrometer (CCS100/M, Thorlabs) at 2 weeks and 36 weeks after the phantom has been fabricated. Prior to each measurement, the spectrometer output was calibrated with a reflectance standard (WS-1-SL, Ocean Optics).

\subsection{Validation of a quantitative fluorescence imaging system}

To provide a preliminary indication of the potential of the developed phantom for imaging system validation, the composite phantom was measured using an in-house built quantitative fluorescence imaging system (Figure 3). The details of the imaging system can be found in work of Xie et al. [13]. Briefly, the system employed two light sources of a white-light LED (MCWHF1, Thorlabs) and a 405-nm LED (M405FP1, Thorlabs) for fluorescence excitation and a highly sensitive CMOS camera (ORCA-Flash4.0, Hamamatsu Photonics) for detection. A liquid crystal tunable filter (LCTF) (VariSpecVIS, PerkinElmer) was used as a bandpass filter that allowed a selected wavelength of light to transmit into the camera to perform multispectral imaging. The concentration of quantum dot was estimated using an isotropic total variation (TV) L1-norm regularized least-squares optimization algorithm [13].

(a) Wide-field spectrally-resolved fluorescence imaging system
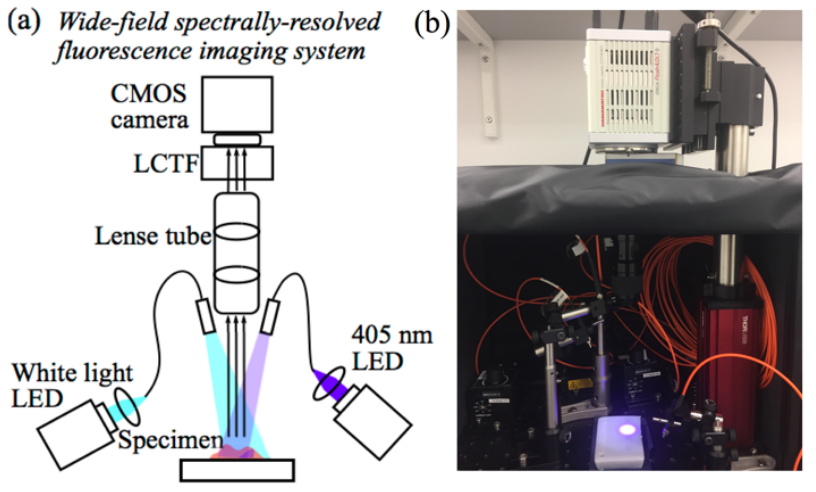

Figure 3: (a) Schematic of the imaging system [13]. (b) Experiment setup of validation using the composite phantom. 

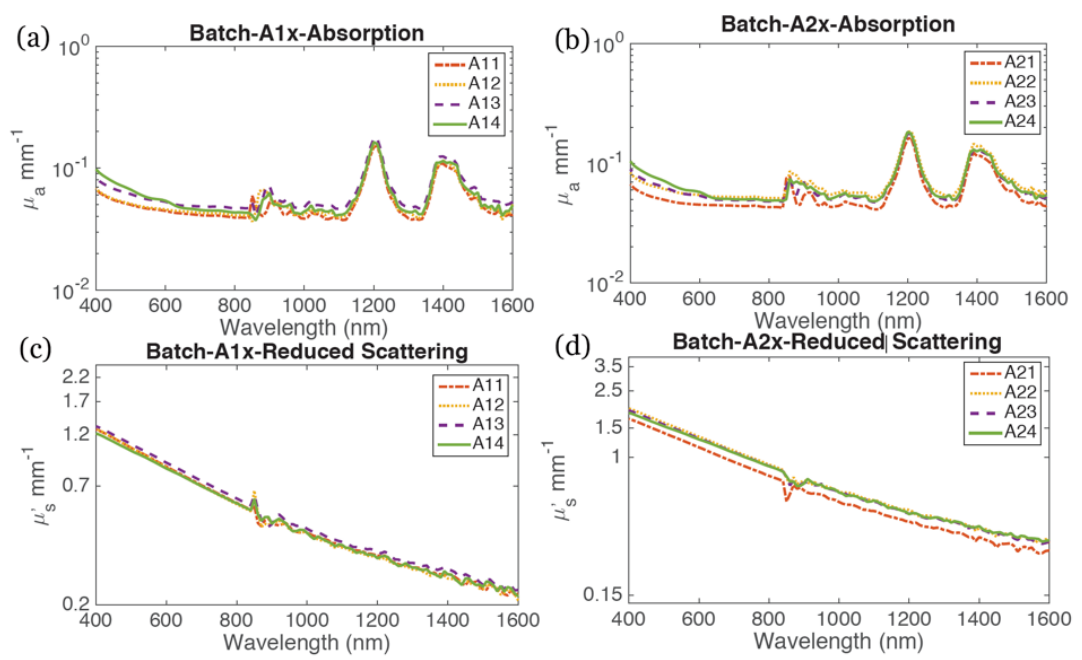

Figure 4. Optical absorption and reduced scattering spectra of the phantom batches with varying titanium dioxide and quantum dots concentrations. Optical absorption, $\mu_{\mathrm{a}}$; Optical reduced scattering, $\mu_{\mathrm{s}}$

\section{RESULTS AND DISCUSSION}

The determined $\mu_{\mathrm{a}}$ and $\mu_{\mathrm{s}}$ ' spectra of the phantom batches with varying titanium dioxide and quantum dots concentrations are shown in Figure 4. In the visible wavelength range $(400-650 \mathrm{~nm})$, the $\mu_{\mathrm{a}}$ spectra for all the batches were dominated by the excitation spectrum of the quantum dots. The $\mu_{\mathrm{a}}$ value increased with the increase of quantum dots concentration. In the near infrared wavelength range $(700-1600 \mathrm{~nm})$, the $\mu_{\mathrm{a}}$ spectra of all the phantom slabs were mainly contributed by the intrinsic optical absorption of gel wax, with three absorption peaks at 930nm, $1210 \mathrm{~nm}, 1450 \mathrm{~nm}$ matching the optical absorption peaks of lipid. The $\mu_{\mathrm{s}}$ ' is solely determined by titanium dioxide; the addition of quantum dots has no effect on the reduced scattering spectrum.

The fluorescence phantoms had good temporal stability in terms of the fluorescence signal intensity overall measurement duration of 34 weeks (Figure 5). The highest change on intensity of $22 \%$ was found in batch A13 and the lowest change of $1 \%$ was found in batch A21. Also the linearity between fluorescence intensity and QDs concentration retained over time.
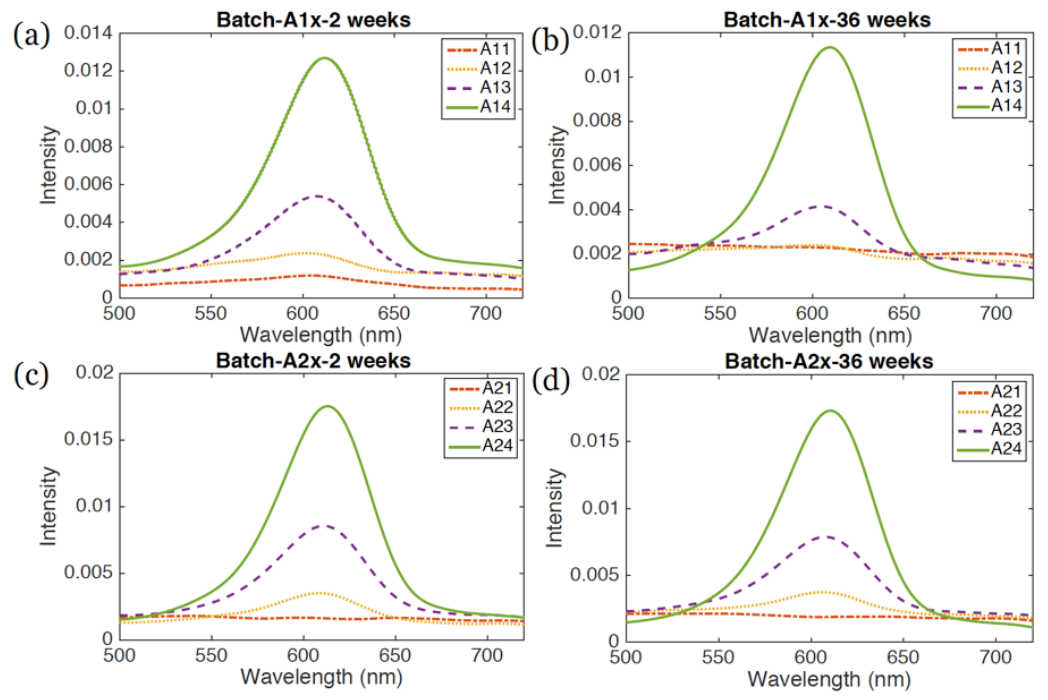

Figure 5. Optical absorption and reduced scattering spectra of the phantom batches with varying titanium dioxide and quantum dots concentrations. Optical absorption, $\mu_{\mathrm{a}}$; Optical reduced scattering, $\mu_{\mathrm{s}}{ }^{\prime}$ 
Results of fluorescence imaging of the developed composite phantoms are shown in Figure 6. The image intensity increased with the increase of the quantum dots concentration. The estimated quantum dots concentrations had good agreement with the concentrations derived from the phantom fabrication process for batches A21 and A22. However, the estimated quantum dots concentration values for batches A23 and A24 were higher than those calculated from fabrication process.
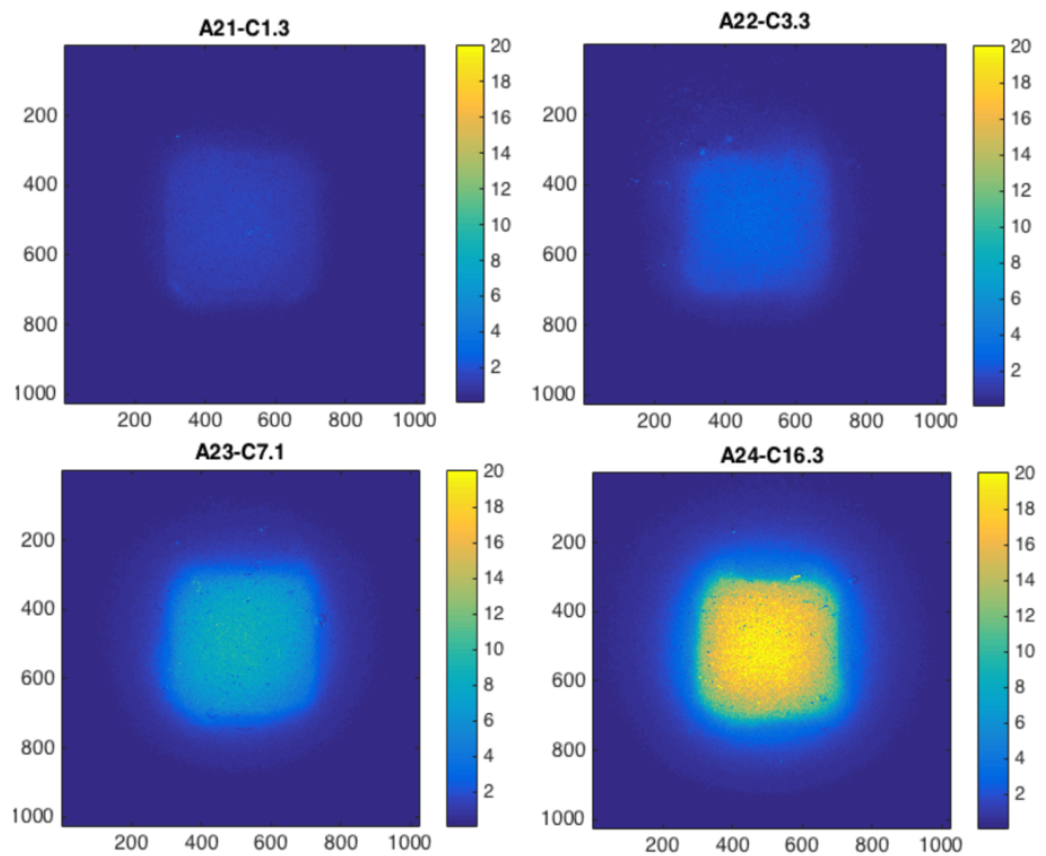

Figure 6. Fluorescence images of composite phantom batches. The estimated quantum dots concentrations for batch A21,

A22, A23, and A24 were $0.013 \%, 0.033 \%, 0.071 \%$ and $0.16 \%(\mathrm{v} / \mathrm{v})$, respectively.

In addition, we moulded the material to a brain-shaped phantom with fluorescence quantum dots inclusion (shown with arrows in Figure 7) to resemble the brain tumour with PpIX as in the 5-ALA guided resection surgery (Figure 7). The tissue-mimicking phantom has great potential of validation of fluorescence imaging system as well as serving in training neurosurgeons. It is worth to mention that with $3 \mathrm{D}$ printing technology we could potentially print mould to shape this material into any anatomically realistic tissue-simulating phantoms for both validation and training purposes [9].
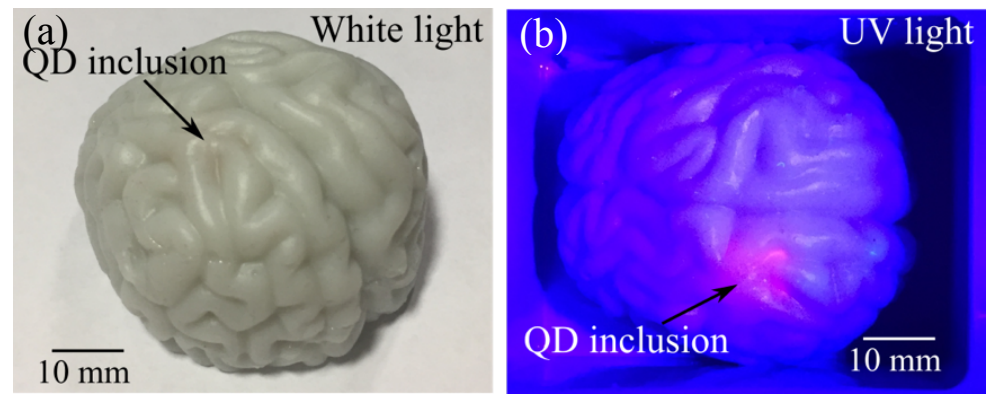

Figure 7: A tissue-mimicking brain phantom manufactured with gel wax presenting under white light (a) and UV light (b) illumination. A chunk material containing QDs embedded within the brain phantom is emitting vivid pink color under UV excitation (b), which resembles the scenario of 5-ALA guided brain tumour resection.

\section{CONCLUSIONS}

We conclude that with good temporal stability, mechanical robustness, and tunable optical properties, gel wax and quantum dots based tissue mimicking phantoms could be useful for standardised performance evaluation of fluorescence imaging devices for guiding a variety of surgical procedures. 


\section{ACKNOWLEDGEMENTS}

This work was supported by Wellcome Trust and the Engineering and Physical Sciences Research Council (EPSRC), 203148/Z/16/Z; NS/A000049/1; 203145Z/16/Z; NS/A000050/1. T Vercauteren is supported by a Medtronic / Royal Academy of Engineering Research Chair in "Machine Learning for Computer-assisted Neurosurgery" [RCSRF1819 $7 \backslash 34$ ].

\section{REFERENCES}

[1] Valdés P.A., Leblond F., Kim A., Wilson B.C., Paulsen K.D., and Roberts D.W., "A spectrally constrained dual-band normalization technique for protoporphyrin IX quantification in fluorescence-guided surgery," Opt. Lett. 37(11), 1817-1819 (2012).

[2] Hardesty D.A. and Sanai N., "The value of glioma extent of resection in the modern neurosurgical era," Front. in Neurol. 2, 140 (2012).

[3] Puppa A.D., Ciccarino P., Lombardi G., Rolma G., Cecchin D., and Rossetto M., "5-aminolevulinic acid fluorescence in high grade glioma surgery: Surgical outcome, intraoperative findings, and fluorescence patterns," BioMed Res. Int. 2014, 232561(2014)

[4] Marois M., Bravo J.J., Davis S.C., Kanick S.C., "Characterization and standardization of tissue-simulating protoporphyrin IX optical phantoms. " J. of Biomedical Optics 21(3), 035003 (2016).

[5] Anastasopoulou M., Koch M., Gorpas D., Karlas A., Klemm U., Garcia-Allende P.B., Ntziachristos V., "Comprehensive phantom for interventional fluorescence molecular imaging, " Journal of Biomedical Optics. 21(9), 091309 (2016).

[6] Pogue B.W., Zhu T.C., Ntziachristos V., Paulsen K.D., Wilson B.C., Pfefer J., Nordstrom R.J., Litorja M., Wabnitz H., Chen Y., Gioux S., "Fluorescence-guided surgery and intervention-An AAPM emerging technology blue paper, " Medical Physics. (2018).

[7] Cook J.R., Bouchard R.R., Emelianov S.Y., "Tissue-mimicking phantoms for photoacoustic and ultrasonic imaging, " Biomedical Optics Express. 2(11), 3193-206 (2011).

[8] Hebden J.C., Hall D.J., Firbank M., Delpy D.T., "Time-resolved optical imaging of a solid tissue-equivalent phantom, " Applied Optics 34(34), 8038-47 (1995).

[9] Maneas E., Xia W., Nikitichev D.I., Daher B., Manimaran M., Wong R.Y., Chang C.W., Rahmani B., Capelli C., Schievano S., Burriesci G., Ourselin S., David A., Finlay M., West S.J., Vercanteren T., Desjardins A.E., "Anatomically realistic ultrasound phantoms using gel wax with 3D printed moulds, " Physics in Medicine \& Biology 63(1), 015033 (2018).

[10] Maneas E., Xia W., Ogunlade O., Fonseca M., Nikitichev D.I., David A.L., West S.J., Ourselin S., Hebden J.C., Vercauteren T., Desjardins A.E., "Gel wax-based tissue-mimicking phantoms for multispectral photoacoustic imaging, " Biomedical Optics Express 9(3), 1151-63 (2018).

[11] Michalet X., Pinaud F.F., Bentolila L.A., Tsay J.M., Doose S., Li J.J., Sundaresan G., Wu A.M., Gambhir S.S., and Weiss S., "Quantum Dots for Live Cells, in Vivo Imaging, and Diagnostics, " Science 307(5709), 538-544(2005).

[12] Prahl S.A., van Gemert M.J., Welch A.J., "Determining the optical properties of turbid media by using the addingdoubling method, " Applied Optics 32(4), 559-568 (1993).

[13]Xie Y., Thom M., Ebner M., Wykes V., Desjardins A., Miserocchi A., Ourselin S., McEvoy A.W., Vercauteren T., "Wide-field spectrally resolved quantitative fluorescence imaging system: toward neurosurgical guidance in glioma resection, " Journal of Biomedical Optics 22(11), 116006 (2017). 